Research Article

Przemystaw Jaszak*

\title{
Optimized design of a semimetal gasket operating in flange-bolted joints
}

https://doi.org/10.1515/eng-2021-0004

Received Apr 22, 2020; accepted Sep 16, 2020

\begin{abstract}
The paper presents a new design of semimetal gasket, which has been subjected to the optimization process. The main objective function was to achieve the maximum elastic recovery of the gasket at imposed functional and strength limitations. The objective function was described indirectly in the form of the required minimum angle of the gasket's unload curve inclination, which was a measure of the average value of the elastic modulus at unloading. Strength limitations resulted from not exceeding the ultimate stress of the materials, while functional limitations imposed the achievement of proper contact pressure that ensures a given level of tightness class. The characteristic dimensions describing the cross-section of the gasket's core were described by five descriptive parameters. As a result of the interrelationship of these parameters, 210 construction models were created that were subjected to compression simulations. This problem was solved using the Finite Element Method. The variant of the gasket fulfilling the imposed limitations and the required minimum objective function was tested experimentally.
\end{abstract}

Keywords: gasket, flange-bolted joints, optimization, FEM, tightness, elastic recovery

\section{Introduction}

The condition for a proper function of any pressure installation is that the medium stored in tanks or transported in pipelines cannot get out of them. Uncontrolled losses of medium, apart from having negative effects on the environment [1-3], also lead to huge financial costs caused by renovations and downtime. Depending on the purpose, each pressure installation has a defined acceptable level of leakage or, which is a more precise definition, can oper- ate at a permissible tightness class. The tightness class defines the permissible leakage level of the sealed medium, referred to as the nominal dimension of the gasket. The basic unit is $\mathrm{mg} / \mathrm{m} \cdot \mathrm{s}$. In accordance with the EN 13555 standard, three basic tightness classes have been defined in pressure installations: L1.0, L0.1 and L0.01. The numerical designation of a given class corresponds to the permissible value of leakage emitted by a given sealing node (calculated in grams per second) in relation to the average diameter of the gasket. The higher the class, the lower emissions to the atmosphere. A flange-bolted joint is the most popular sealing node in many pressure installations. It is characterized by the fact that by increasing the mounting load of the bolts, the level of leakage decreases. Nevertheless, there is a certain limit, which is determined by the resistance of the weakest elements of such a node [4]. The weakest element is usually the gasket. Its strength limit is mainly connected to increased plasticity. Greater plasticity of the gasket causes it to have lower strength, and vice versa. The analysis of the impact of the gasket's elasticity on the leakage level from the flange-bolted joint was presented, among others, in [5-8]. The most popular solutions of the gaskets used in medium and high-pressure installations are the so-called semimetal gaskets. The best known include the spiral wound gasket (SWG) and the serrated gasket (SG). Each of them generates different operational problems. One of the most important problems of spiral wound gaskets is the inward buckling of metal windings at an excessive compressing load [9-12]. In the case of serrated gaskets, an excessive load can lead to cracking of the metal core [13] and an uneven distribution of the contact sealing surface [7]. This work analyzes the construction of a new solution for the semimetal gasket. The purpose of this analysis was to determine an optimal shape of the metal core, which will consequently translate into maintaining a high level of tightness class.

\footnotetext{
^Corresponding Author: Przemysław Jaszak: Wroclaw University of Science and Technology, Department of Mechanical and Power Engineering, Wybrzeże Wyspiańskiego 27, 50-370 Wroclaw, Poland; Email: przemyslaw.jaszak@pwr.edu.pl
}

๖ Open Access. ๑) 2021 P. Jaszak, published by De Gruyter.

(cc) BY License 


\section{Research object and scope of work}

The subject of the study was a semimetal gasket, the construction of which is shown in Figure 1. According to the original concept [14], this gasket consists of a metal core with a cross-section resembling the letter " $\mathrm{X}$ ", and a flexible material located in grooves cut on the faces of this core.

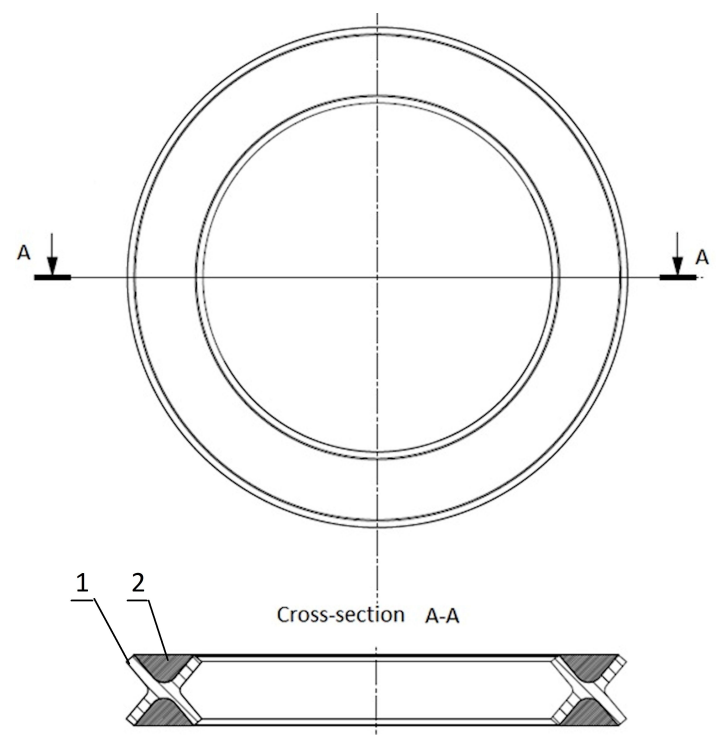

Figure 1: The semimetal gasket with an elastic metal core, 1 - metal core, 2 - flexible material

The elastic material filling the metal core can be expanded graphite, PTFE or expanded mica. In this work, expanded graphite was adopted as the flexible material, while steel 1.4301 was used to make the metal core. The purpose of the numerical analysis conducted in the first part of this paper was to determine the optimal dimensions that describe the geometry of the cross-section of the metal core. The optimal solution was one with high elasticity and high elastic recovery. As a result, such a solution should translate into a high tightness class. At the final stage of the work, an experimental verification of the optimal solution was carried out. The experiment assumed the determination of the characteristics describing the elastic-plastic properties and the characteristics of the tightness.

\section{Formulation of the optimization problem}

The optimal design of the gasket used in flange-bolted joints is one that should be characterized by:

- high tightness at low assembly loads,

- high elasticity,

- high mechanical strength,

- high corrosion resistance,

- high resistance to creep and relaxation.

All these properties are associated with the selection of appropriate materials. In a typical semimetal gasket, the metal element usually provides elasticity and strength, whereas the flexible material (due to its high plasticity) provides leakage proofness. In the case where a sealing node is subjected to an alternating load (temperature, pressure, mechanical vibrations), the level of tightness is determined by another gasket parameter, i.e. elastic recovery. The elastic recovery (in relation to the function of the sealing in the flange-bolted joint) can be characterized as the ability to maintain the highest possible contact pressure with a decreasing load on the bolts. An impact of the gasket's elasticity on the load distribution and elastic recovery in the joint's components is shown in Figure 2.

Assuming that bolts deform elastically throughout the entire load range and that the stiffness of the compressed elements (flanges, nuts, washers and gaskets) can be represented by one of the non-linear gasket's characteristics, then the assembly state of the joint can be represented by means of the chart stiffness shown in Figure 2a). The common point of the bolt's characteristics and gasket's characteristics is determined by assembly force $\left(F_{A}\right)$. In the case of an operating load, the pressure of the sealed medium acting on the blind ends (covers, bends) causes hydrostatic force $\left(F_{H}\right)$ - see Figure $2 \mathrm{~b}$ ). As a result, this force causes the increase of the bolt's load $\left(F_{B}\right)$, whereas the gasket's load (deformation) decreases to the residual clamping force $\left(\mathrm{F}_{R}\right)$. The smaller the residual clamping force, the smaller the tightness of the joint. A proportion of the hydrostatic force, which increases the bolt's load $\left(\mathrm{F}_{H}^{\prime}\right)$ and decreases the residual clamping force $\left(\mathrm{F}_{H}^{\prime \prime}\right)$, mainly depends on the stiffness characteristics of the bolt and gasket. The best solution, from the point of view of the joint's operation and the maintaining of a high residual clamping force, is one where bolts are very stiff and the gasket is resilient. Nonetheless, the chart presented in Figure 2b) does not include the gasket's plastic deformation. The example where the gasket was deformed plastically is presented in Figure 2c). In such a case, the hydrostatic force 

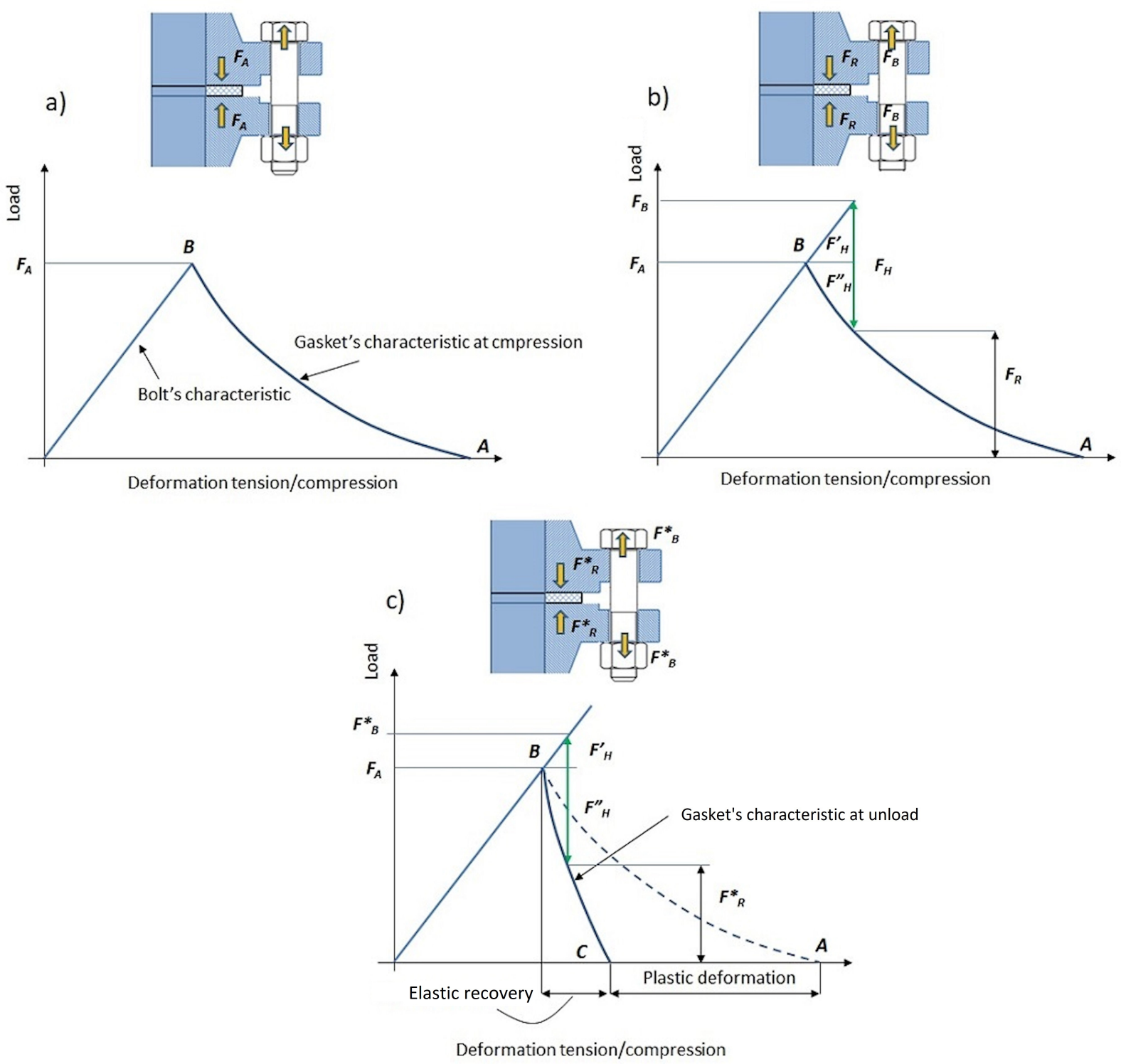

Figure 2: The influence of the stiffness of the joint's elements on the residual force

spreads between the bolt's stiffness line and the gasket's $\mathrm{BC}$ curve. The $\mathrm{BC}$ curve is inclined at a larger angle to the abscissa than the $\mathrm{AB}$ curve. The most advantageous, from the point of view of maintaining a high residual clamping force, would be the case where the BC curve is inclined at the smallest possible angle to the abscissa, and where its deviation from the $\mathrm{AB}$ curve is as small as possible. A gasket fulfilling these requirements should have high elasticity and high elastic recovery. Based on the above analysis, it was found that a basic objective function of the optimal gasket design is one which has low flexibility for the unloading process. This parameter can be indirectly determined by the angle of inclination of the unloading $\mathrm{BC}$ curve relative to the abscissa of the coordinate system. In the analyzed solution, the parameters that limit the sought area of the optimal gasket's structure were the geometrical dimensions of the core's cross-section, the strength of the used materials, as well as the ensuring of a minimum pressure exerted on the sealing surface. A similar optimization model was presented in [15]. The limitations of the gasket's dimensions were the maximum outer diameter $\mathrm{d}_{z}=$ $90 \mathrm{~mm}$, the minimum inner diameter $\mathrm{d}_{w}=49 \mathrm{~mm}$, and the maximum height $\mathrm{h}=10 \mathrm{~mm}$. Characteristic dimensions describing the shape of the gasket's cross-section (adopted as decision parameters of the optimization apparatus) are presented in Figure 3. The set of decision parameters was 
described as:

$$
\begin{aligned}
& s\left\{R_{1} ; R_{2} ; \alpha ; t ; b\right\} \\
& R_{1} \in\{2 ; 3 ; 4 ; 5 ; 6\} ; R_{2} \in\{0.5 ; 1 ; 2 ; 3 ; 4 ; 6\} ; \\
& \alpha \in\{65 ; 75\} ; t \in\{0.7 ; 1\} ; \\
& b \in\{8 ; 10 ; 12 ; 14 ; 16 ; 18\}
\end{aligned}
$$

where $R_{1}(\mathrm{~mm})$ was the rounding radius of the bottom in the front groove, $R_{2}(\mathrm{~mm})$ was the rounding radius of the bottom in the lateral grooves, $\alpha\left(^{\circ}\right)$ was the angle of the lateral grooves, $t(\mathrm{~mm})$ was the thickness of the segment, and $b(\mathrm{~mm})$ was the width of the gasket. The enforcing parameter was the gasket's deformation $U_{Z}=3 \mathrm{~mm}$.

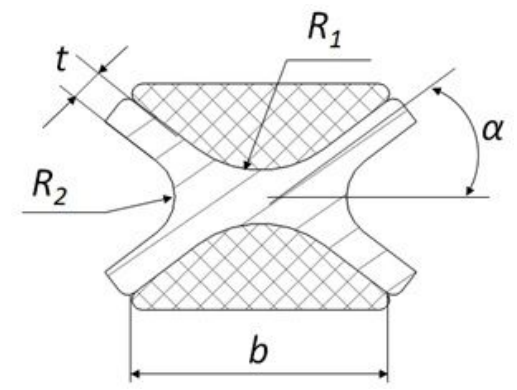

Figure 3: The characteristic dimensions of the gasket

The independent variables were the parameters describing the strength of materials, which determined some limitations of the searched area of the optimal design. The first limitation was the allowable contact pressure of the expanded graphite equal to $180 \mathrm{MPa}$. This limitation was written as:

$$
a\left(s ; u_{z}\right) \leq 180 \mathrm{MPa}
$$

The second limitation involved not exceeding the value of the ultimate stress of the metal core equal to $550 \mathrm{MPa}$ :

$$
b\left(s ; u_{z}\right) \leq 550 \mathrm{MPa}
$$

The third limitation was obtaining the minimum graphite contact pressure $\mathrm{p}_{\min }=10 \mathrm{MPa}$, which ensures the tightness at the level of $0.1 \mathrm{mg} / \mathrm{sm}$. This limitation can be written as:

$$
c\left(s ; u_{z}\right) \geq 10 \mathrm{MPa}
$$

A graphic description of the optimization process is shown in Figure 4. Based on the above objective function and the imposed limitations, the objective functions can be presented as follows:

$$
\left\{\begin{array}{l}
E_{G}\left(s ; u_{z}\right)=\min \\
\lim \left\{\begin{array}{l}
a\left(s ; u_{z}\right) \leq 180 \mathrm{MPa} \\
b\left(s, u_{z}\right) \leq 550 \mathrm{MPa} \\
c\left(s ; u_{z}\right) \geq 10 \mathrm{MPa}
\end{array}\right.
\end{array}\right.
$$

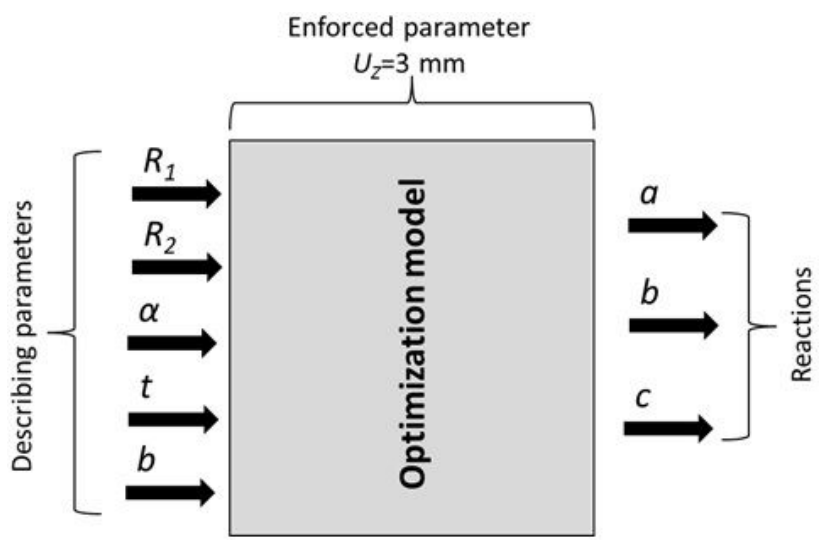

Figure 4: Model of the gasket's optimization

Based on the assumed range of the parameters that describe the geometries of the core's cross-section, 210 models of the gasket's design were generated. Examples of the solutions are presented in Figure 5.
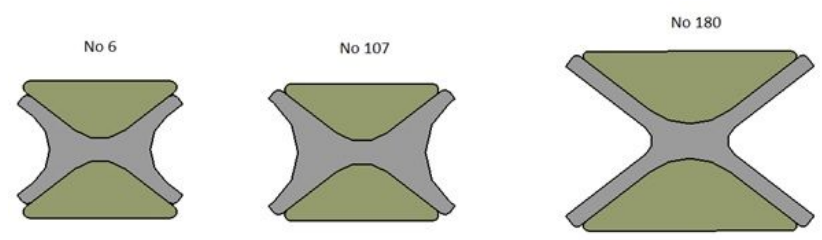

Figure 5: The exemplary variants of the gasket's cross-section

\section{Numerical calculations}

The Finite Element Method was used as a tool to optimize the structure.

Numerical models of the 210 considered variants were prepared as axisymmetric. Each of the numerical models consisted of a gasket located between two plates representing the compression surfaces of a hydraulic press. Higherorder structural finite elements were selected to discretize areas of individual parts. Depending on the gasket's design, the number of finite elements ranged from 3200 to 7300. The mesh density in the expanded graphite was 0.1 $\mathrm{mm}$, whereas in the metal core it was $0.2 \mathrm{~mm}$. The mesh density of the metal plates ranged from $0.1 \mathrm{~mm}$ to $1 \mathrm{~mm}$. The discretization of the exemplary model is shown in Figure 6a). Each contacting surface was set as frictional. The friction coefficient on the contact area between the metalgraphite was 0.4 , while the metal-metal one was 0.2 . The boundary conditions of the model were: the fixing of the 
a)

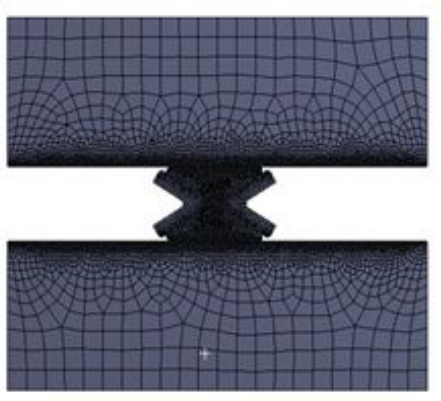

b)

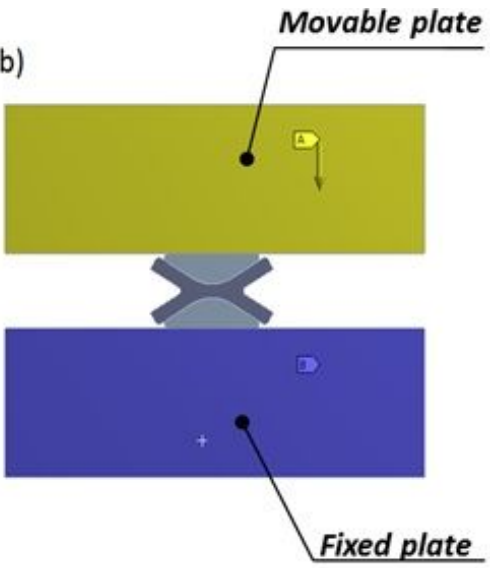

Figure 6: The numerical model of the gasket's compression: a) discretization of particular areas, b) boundary conditions

a)

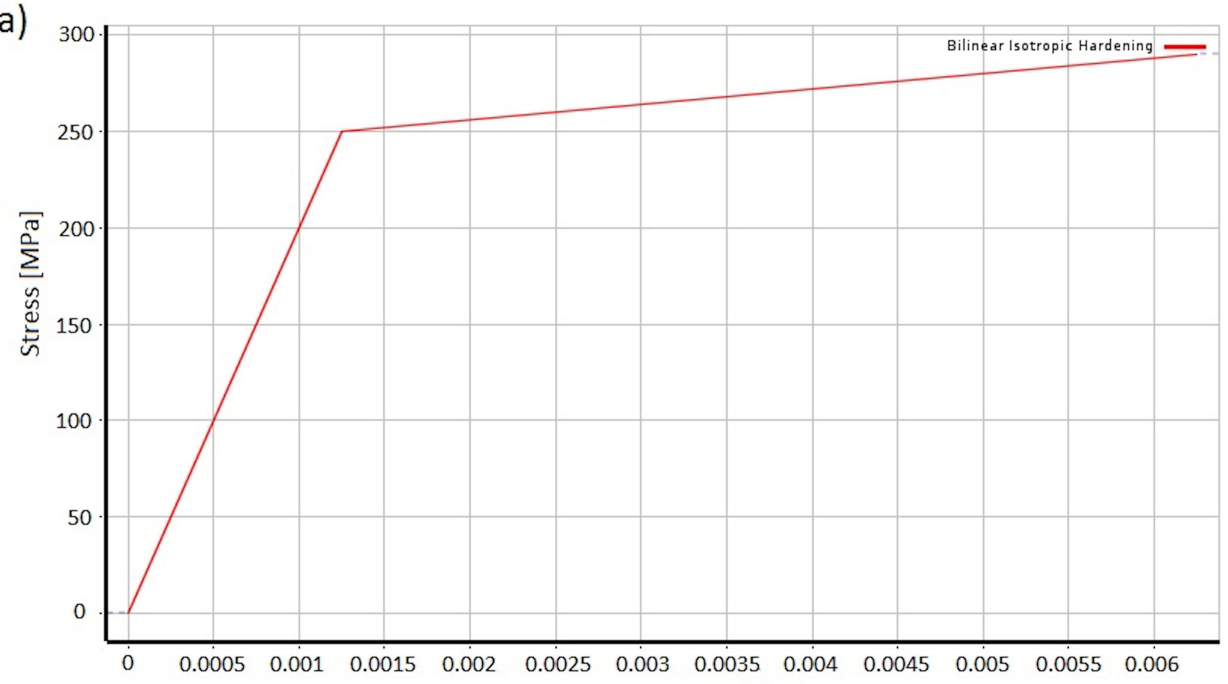

b)

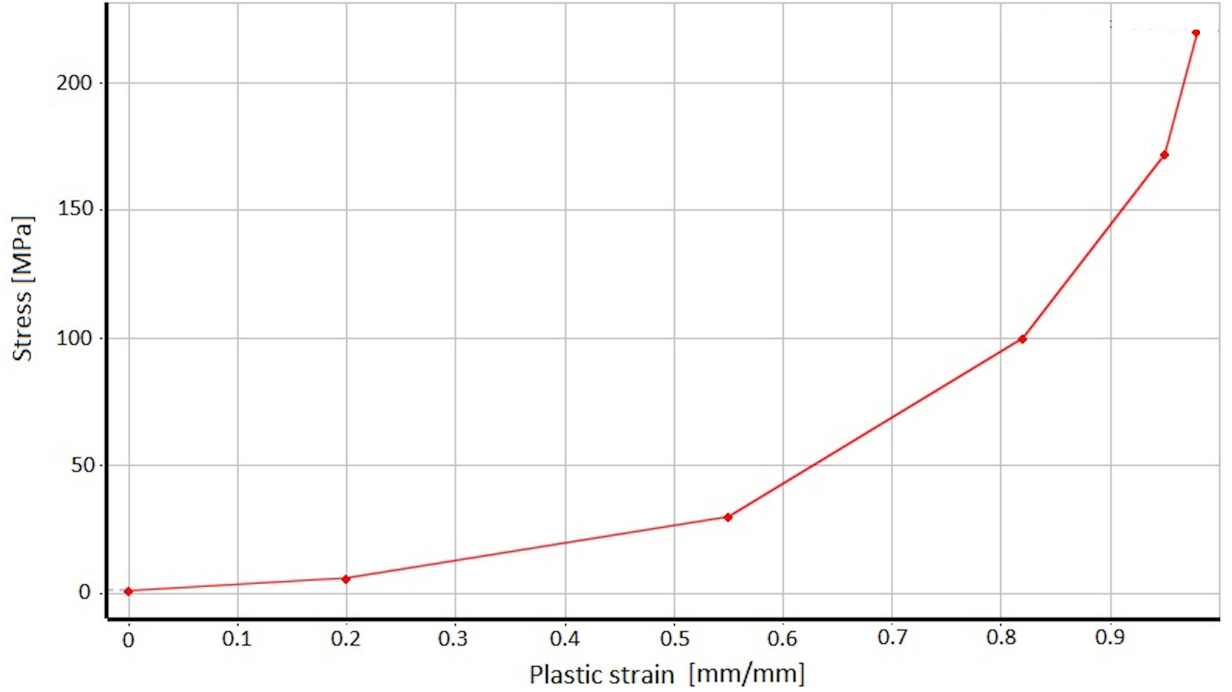

Figure 7: Characteristics of the materials: a) isotropic bilinear model of steel, b) isotropic multilinear model of expanded graphite 
lower plate - preventing its displacement in the axial direction, and the displacement of the upper plate by $U_{Z}=$ $3 \mathrm{~mm}$ - realizing the compression process of the gasket Figure 6b).

The model representing metal parts was an isotropic, bilinear elasto-plastic one in which the value of elasticity modulus was $E=206000 \mathrm{MPa}$, Poisson's ratio $v=0.3$, and the material strengthening module in the plastic zone $E_{U}$ $=8000 \mathrm{MPa}-$ see Figure 7a). To reflect the properties of the expanded graphite, an isotropic, multi-linear material model was adopted. Its characteristics (being the result of experimental measurements) are shown in Figure 7b). This, of course, was a simplification of the graphite's properties, since this material exhibits high anisotropy at a high compression level. a)

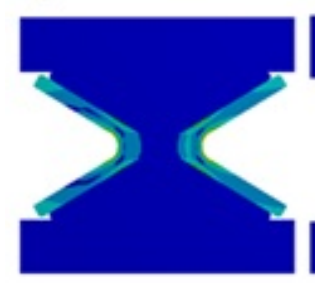

b)
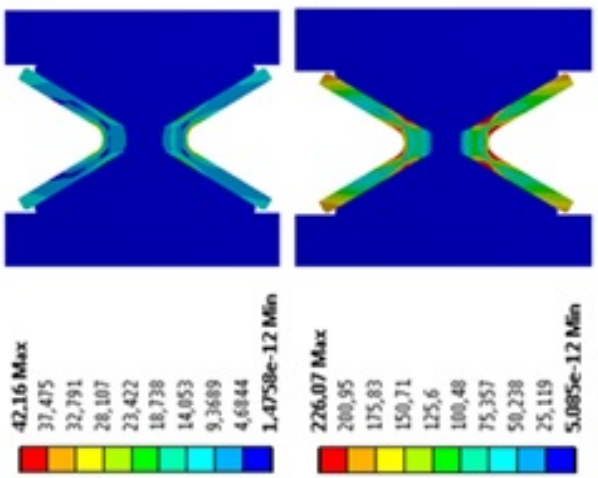

\subsection{Numerical results}

Figure 8 shows the visualization of the compression process of gasket model No. 180. This variant met only two lim-

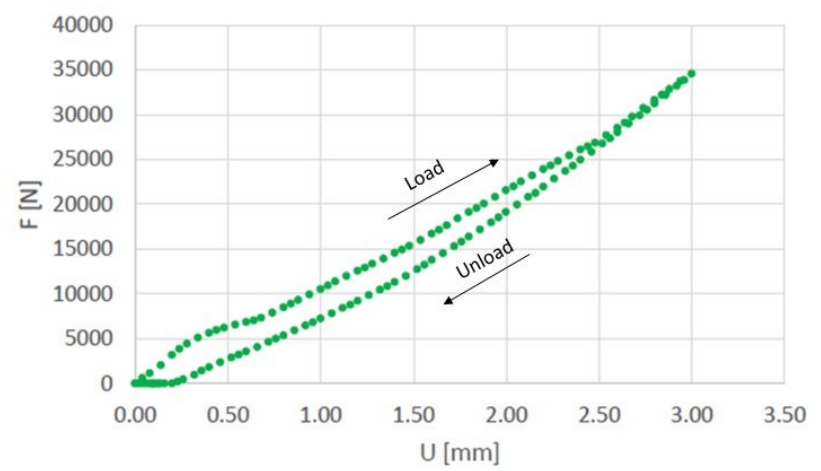

Figure 10: Compression characteristics of gasket variant No. 110
Figure 8: Equivalent stress distribution in MPa of gasket model No. 180: a) compression $0.3 \mathrm{~mm}$, b) compression $0.5 \mathrm{~mm}$, c) compression $1.5 \mathrm{~mm}$, d) compression $3 \mathrm{~mm}$, e) decompression $1.5 \mathrm{~mm}$ a)

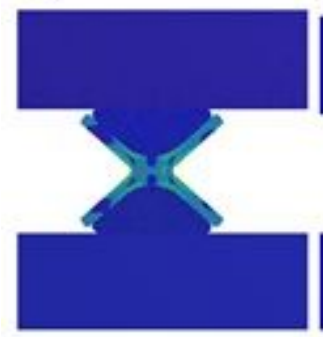

b)
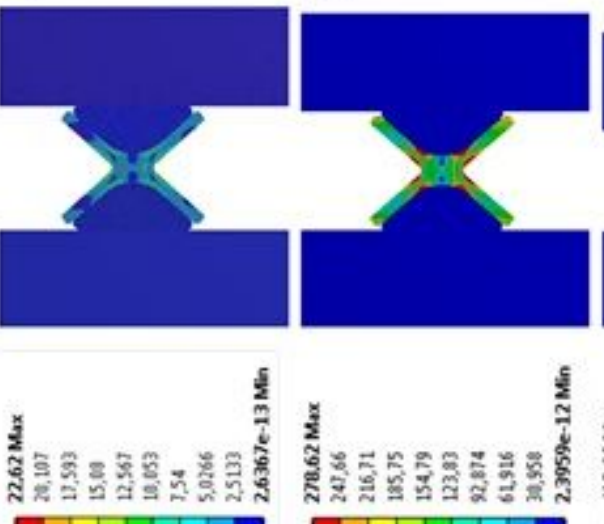

c)
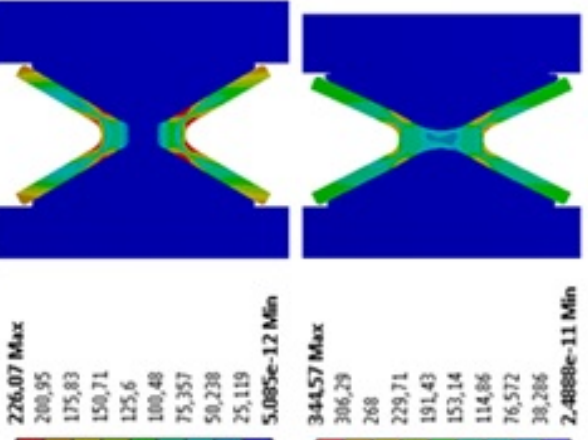

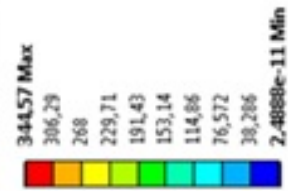

d)

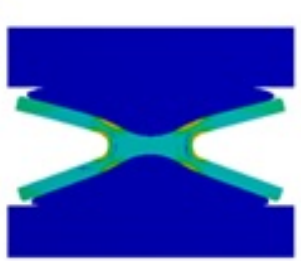

e)
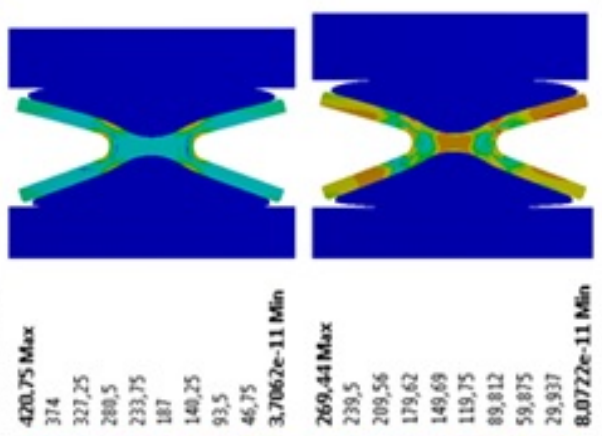
(6)

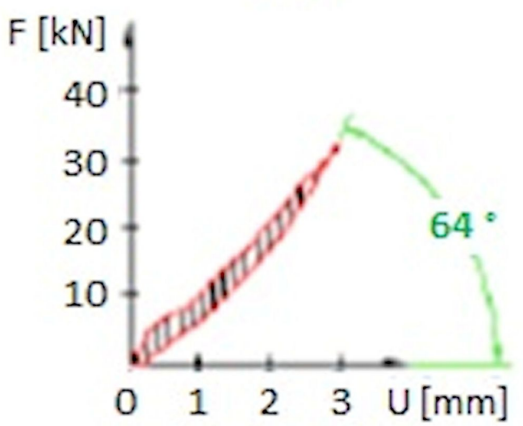

(96)

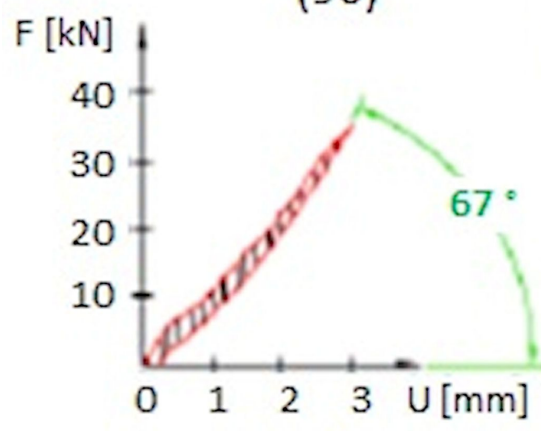

(7)

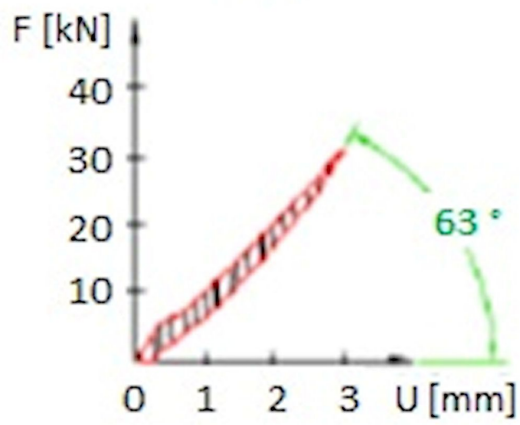

(107)
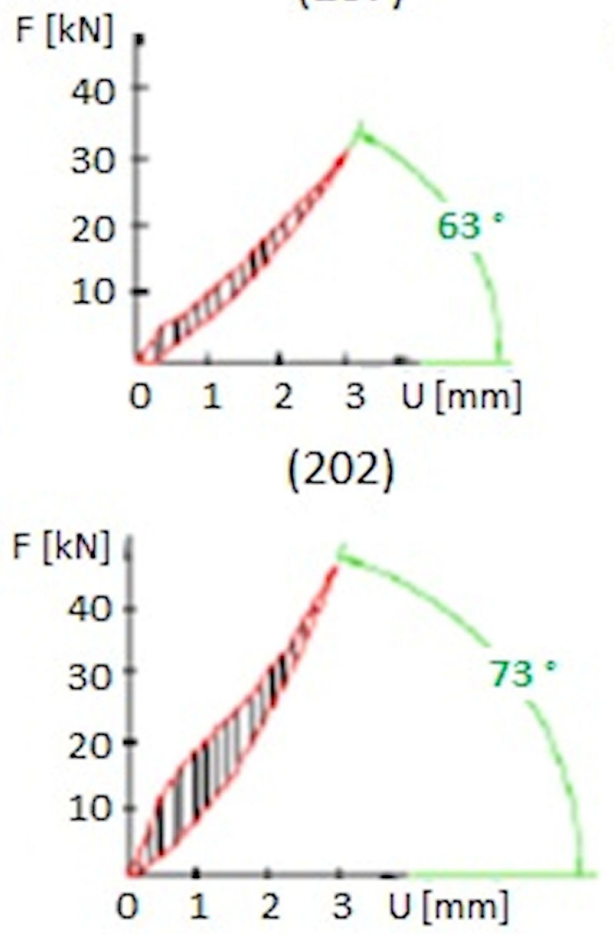

(93)

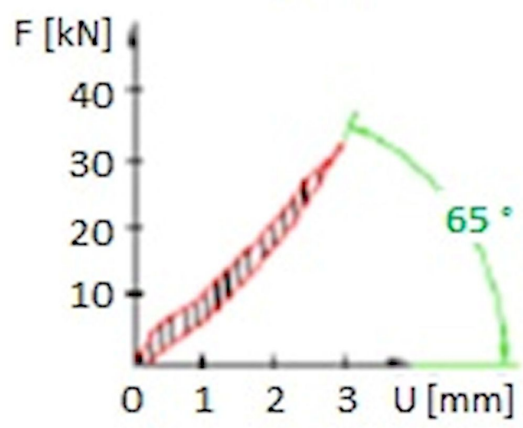

(110)

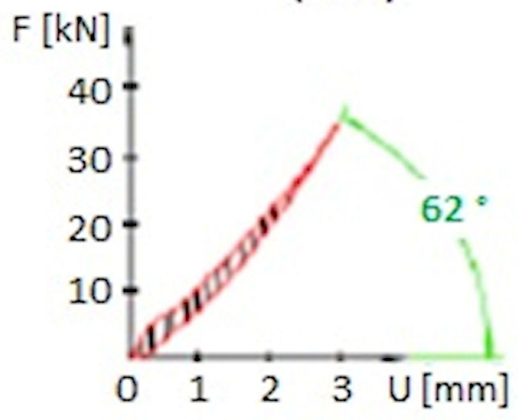

Figure 11: The stiffness characteristics of the selected gasket's variants that met all the limitations

itations concerning the strength of the materials. At a compression of $U_{Z}=3 \mathrm{~mm}$, the value of the maximum stress in the metal core was $S_{\max }=420.7 \mathrm{MPa}$, and the maximum stress in the graphite was Smax $=18.8 \mathrm{MPa}$. However, this variant did not meet the limitation resulting from the minimum contact pressure. The contact pressure on the gasket's surface at a maximum compression $\mathrm{U}_{Z}=3 \mathrm{~mm}$ was 4.3 MPa. Based on the numerical calculations, only 7 out of 210 gasket variants met all the imposed limitations.

Figure 9 presents the compression process of variant No. 110. The stress in the metal core at a compression of 3 $\mathrm{mm}$ was $S_{\max }=507.8 \mathrm{MPa}$, the stress in the graphite was $S_{\max }=48.2 \mathrm{MPa}$, and the contact pressure was $21.3 \mathrm{MPa}$.
Figure 10 presents the deformation characteristics of this variant. The maximum force necessary to deform the gasket by $3 \mathrm{~mm}$ was about $35 \mathrm{kN}$.

The average angle of the unload curve was 62 degrees, and it was the minimum of the objective function. In addition, a small hysteresis can be observed, i.e. the area located between the load and unload curves. A compilation of the stiffness characteristics of the variants that met the imposed limitations is shown in Figure 11. Table 1 contains the values of the inclination angles of the unload curve, parameters $j^{2}$ that determine the relative value of the hysteresis area, and the dimensions of the gasket's cross-section. 
Table 1: Compilation of the average values of the inclination angles of the unload curve, the relative hysteresis surface area, and the characteristic dimensions of the gaskets that met all the limitations

\begin{tabular}{cccccccc}
\hline Variant & Area & $\beta$ & $\mathrm{R}_{1}$ & $\mathrm{R}_{2}$ & $\alpha$ & $\mathrm{b}$ & $\mathrm{t}$ \\
\hline No & $\mathrm{j}^{2}$ & $\mathrm{deg}$ & $\mathrm{mm}$ & $\mathrm{mm}$ & $\circ$ & $\mathrm{mm}$ & $\mathrm{mm}$ \\
6 & 0.964 & 64 & 2 & 2 & 75 & 8 & 0.7 \\
7 & 0.891 & 63 & 2 & 2 & 75 & 10 & 0.7 \\
93 & 0.968 & 65 & 2 & 3 & 75 & 10 & 0.7 \\
96 & 0.85 & 67 & 2 & 1 & 75 & 8 & 0.7 \\
107 & 0.86 & 63 & 2 & 3 & 75 & 10 & 0.7 \\
110 & 0.731 & 62 & 3 & 0.5 & 75 & 10 & 0.7 \\
202 & 1.906 & 73 & 6 & 2 & 65 & 16 & 1 \\
\hline
\end{tabular}

\section{Experimental research}

In order to verify the numerical calculations of the optimal gasket's structure, experimental tests were carried out. In the first part of the experiment, the elastic behavior of the gasket was determined. In the second part, the leakage characteristic of the optimal gasket variant was determined.

\subsection{Elasticity and elastic recovery}

\subsubsection{Test rig}

Figure 12 presents the test stand.

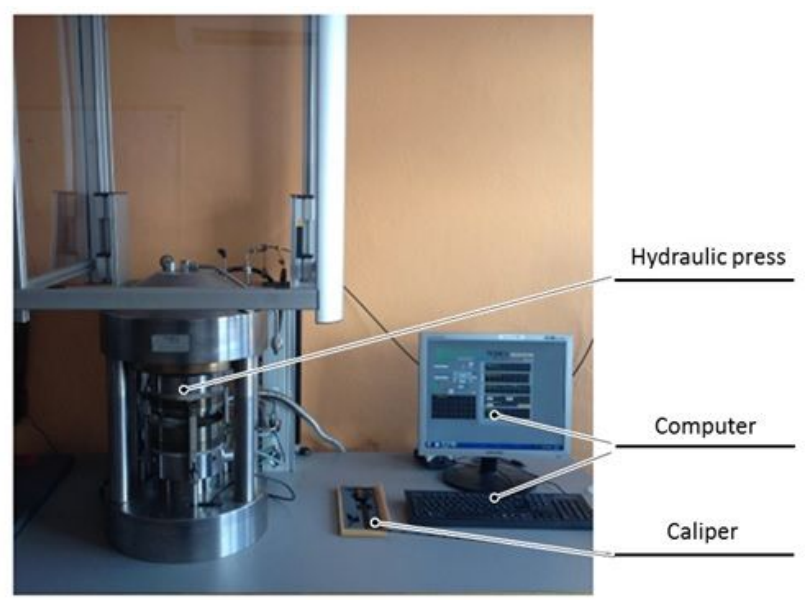

Figure 12: Test rig for the determination of the gasket's elastic properties

The main element was a computer-controlled hydraulic press. The other elements of the test rig included a hydraulic press control system and a computer that records the course of the test. The parameters of the hydraulic press were: the maximum compressive force of 1 $\mathrm{MN}$, the maximum size of the tested gasket (outer diameter) of $170 \mathrm{~mm}$, the maximum operating temperature of 300 C degrees.

\subsubsection{The test procedure}

The procedure was as follows: the gasket was placed in a concentric way on the lower plate of the hydraulic press. The upper plate of the press was set to touch the top of the gasket's surface. Once a contact between the gasket and the upper plate was reached, the maximum compression value $U_{Z}=3 \mathrm{~mm}$ was set. Due to displacement sensors mounted between the upper and lower plates, as well as an extensometer in the lower plate, the dependence between the compressing force and the gasket's displacement was recorded.

\subsubsection{Test results}

Figure 13 presents the experimentally determined deformation characteristics of the tested gasket together with the characteristics obtained by means of numerical calculations. It can be stated that despite simplifications adopted in the numerical model of the graphite, the obtained characteristics did not differ significantly from the experimental characteristics. The angle of inclination of the unloading curve was 65 degrees, while the force causing the compression of $3 \mathrm{~mm}$ was $38 \mathrm{kN}$. The sample of the gasket after the compression test is shown in Figure 14. 


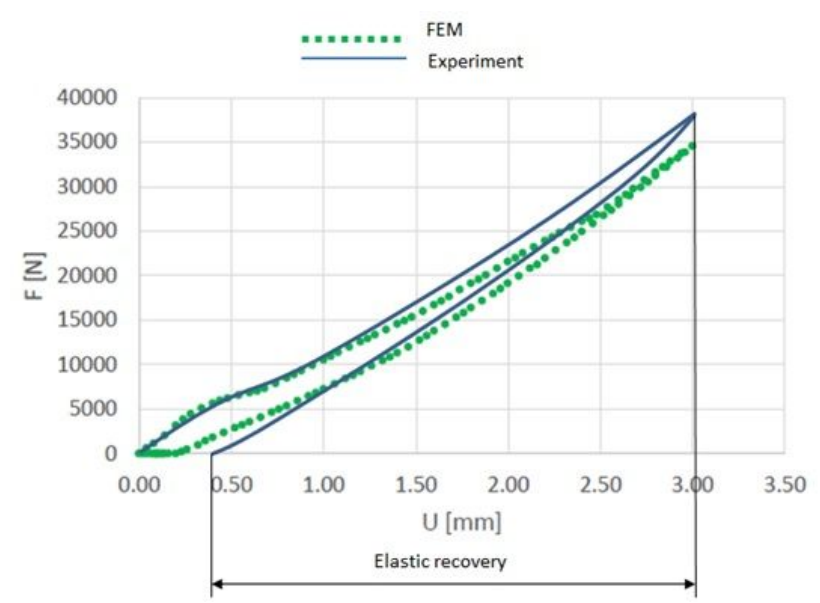

Figure 13: Characteristics of the gasket's stiffness

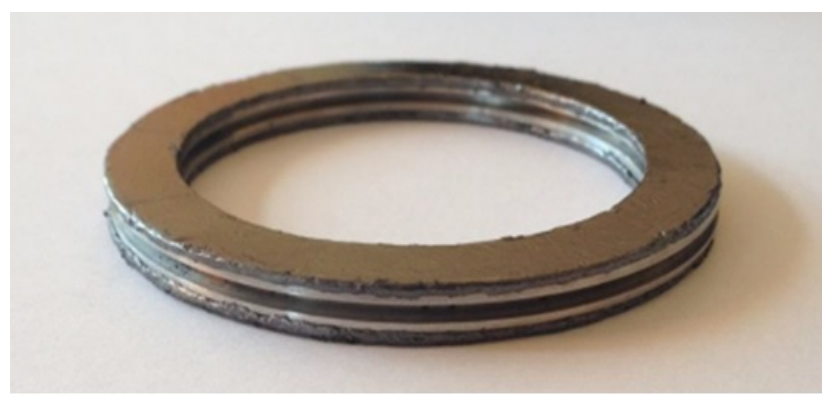

Figure 14: Gasket after the compression test

\subsection{Leakage level}

In order to confirm the optimal gasket solution, a leakage test was performed. To reflect the real operating conditions of the gasket, the test was carried out on a flange-bolted joint.

\subsubsection{Test rig}

The test stand presented in Figure 15 consisted of two flanges with dimensions DN40 and PN40, four M16 bolts with strength class 8.8, a glass cover constituting a collective collector, a helium detector, a helium reservoir, and a vacuum pump connected to the detector.

Each bolt was equipped with a micrometer measurement system, which is shown in Figure 16.

This solution was based on the guidelines included in the EN 28091-2 standard. The idea of such a solution was to measure the length of a metal rod (9). When tightening the nuts (3), the micrometer gauges (1) mounted on the ends of the bolts indicated the current value of the rod protruding above the end of the bolt. Based on such measurement, the elongation of a given bolt can be directly assessed, and after determining the characteristics describing the relationship between the bolt's tensile force and its elongation, the value of its tension can be determined. Exemplary bolt characteristics are shown in Figure 17.
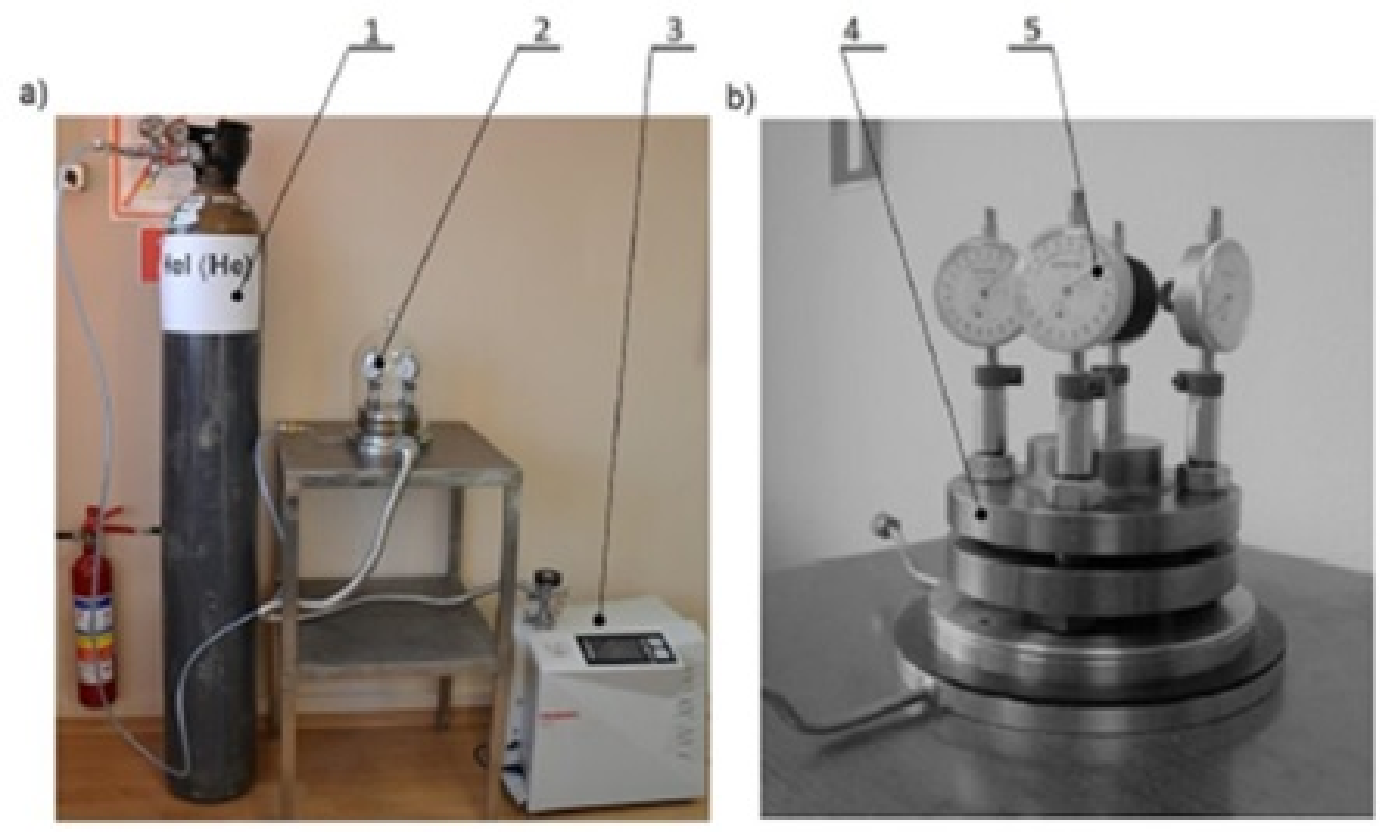

Figure 15: Test rig for the leakage test: 1- helium reservoir, 2-glass cover, 3 - helium detector, 4- flange bolted joint with gasket, 5 micrometric measuring system 


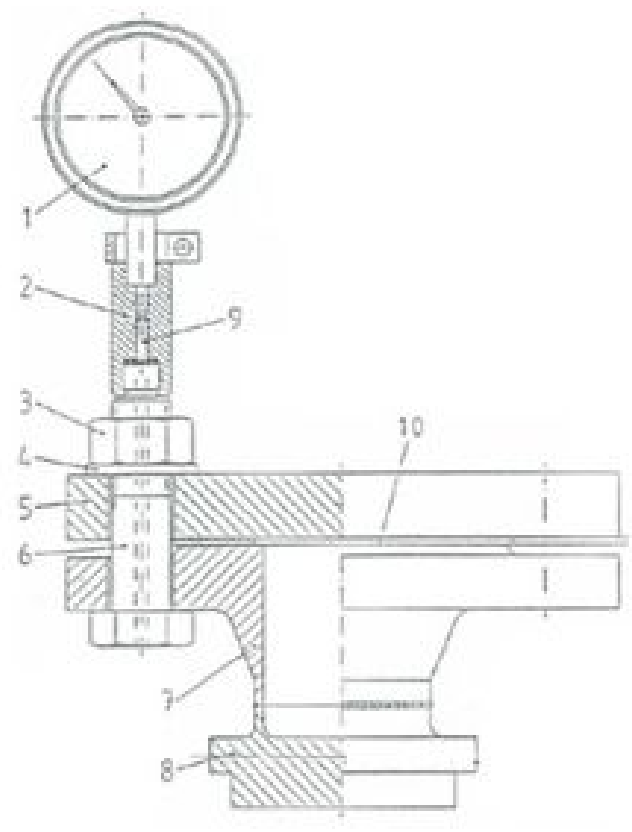

Figure 16: Micrometric system to measure the elongation of bolts

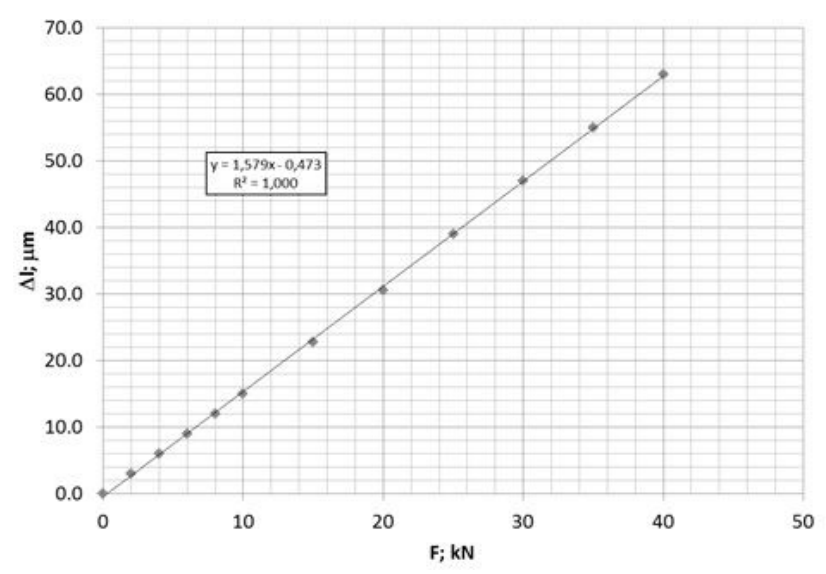

Figure 17: Characteristics describing the dependence of the bolt's elongation in the function of tensile force

\subsubsection{Test procedure}

After placing the gasket on the lower flange, the upper flange was mounted and the nuts were tightened with a small torque. Afterwards, the micrometer sensors were installed. The test consisted of a gradual increase of the bolt's load and then a gradual decrease. Ultimately, four bolt tension values were determined for the loading of 10 , 20,30 and $40 \mathrm{kN}$, and three values for the reduction of the load, i.e. 30, 20 and $10 \mathrm{kN}$. In each load point, 10 bar of helium pressure was applied on the joint, a glass cover was put on the joint and the level of leakage was measured.

\subsubsection{Results}

Figure 18 presents the tightness characteristics of the optimal gasket solution. At the maximum gasket load, i.e. 40 $\mathrm{kN}$, the measured leakage value was $0.0037 \mathrm{mg} / \mathrm{s} \cdot \mathrm{m}$. Very optimistic results of the leakage level were obtained when the load on the bolts was reduced. The slope of the unloading leakage curve was inclined at a small angle to the abscissa. The leakage level during unloading of the gasket's compression at $10 \mathrm{kN}$ was approximately $0.1 \mathrm{mg} / \mathrm{s} \cdot \mathrm{m}$. This effect was due to the high elastic recovery of the metal core and the good plastic properties of the flexible material.

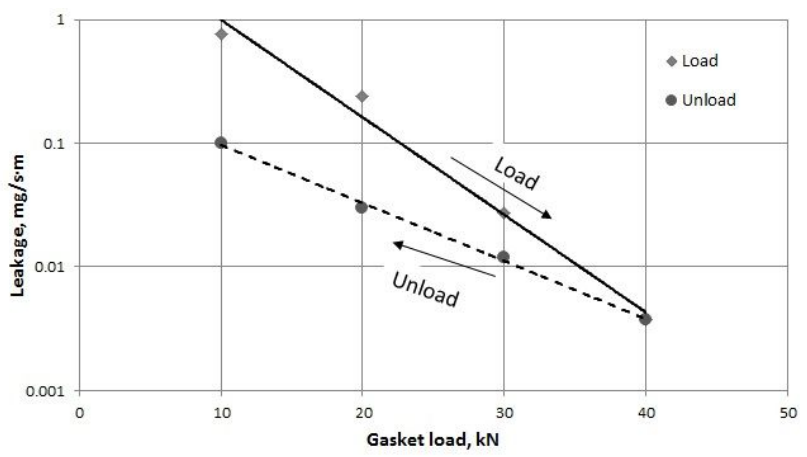

Figure 18: Tightness characteristic of the optimal gasket solution

\section{Conclusions}

Based on the numerical calculations and experimental tests, the results can be concluded in the following way:

- The proper shaping of the metal gasket's core has a major influence on its elasticity, as well as on the amount of absorbed and dissipated energy. The measure of dissipated energy was the area of the hysteresis loop formed between the load and unload gasket curves.

- A greater hysteresis loop causes the unload gasket curve to deviate from the load curve.

- Greater deviations of the unload gasket curve from the load curve cause the elastic recovery to be smaller.

- An excessive increase of the gasket's elasticity leads to an excessive increase of the stress in the metal core.

- As the load on the bolts gradually decreases, the gasket with an increased elastic recovery maintains a high level of tightness of the flange-bolt joint. 
- After the optimization process, only 7 out of 210 gasket solutions were revealed. These solutions met all the imposed limitations. Nonetheless, only gasket design No. 210 exhibited the minimum of the objective function, and therefore this solution has been recognized as the optimal design.

- The optimal gasket design provides a moderate and reduced stress in the expanded graphite $(48.2 \mathrm{MPa})$ and in the metal core $(507.8 \mathrm{MPa})$, but what is more important, the pressure when compressing the gasket to $3 \mathrm{~mm}$ was equal to $21.3 \mathrm{MPa}$.

- The optimal gasket design was experimentally tested. The experimental results showed that the compression characteristics slightly differ from the numerical results. These small deviations can be caused by the adapted isotropic material model of the expanded graphite.

- The leakage test of the optimal gasket showed that under a compression load of $40 \mathrm{kN}$, the gasket provides a leakage level of $0.0037 \mathrm{mg} / \mathrm{s} \cdot \mathrm{m}$. Moreover, during the unloading, the gasket keeps a very low leakage level of approximately $0.1 \mathrm{mg} / \mathrm{s} \cdot \mathrm{m}$.

\section{Summary}

The paper presents a new design of a semimetal gasket, which was subjected to the optimization process. The main objective function was to achieve the maximum elastic recovery of a gasket at imposed functional and strength limitations. The objective function was described indirectly in the form of the required minimum angle of the gasket's unload curve inclination, which was a measure of the average value of the elastic modulus at unloading. Strength limitations resulted from not exceeding the ultimate stress of the materials, while functional limitations imposed the achievement of a proper contact pressure that ensures a given level of tightness class. The geometry of the crosssection of the metal core was determined by five descriptive parameters. As a result of the interrelationship of these parameters, 210 construction models were created and subjected to compression simulations. This problem was solved by means of the Finite Element Method. The gasket variant fulfilling the imposed limitations and the required minimum of objective function was tested experimentally. In the first stage, a compression test was carried out, which allowed the gasket's elasticity and elastic recovery to be determined. In the second stage, a leak test was carried out. At the maximum gasket load of $40 \mathrm{kN}$, the tightness level was higher than $0.01 \mathrm{mg} / \mathrm{s} \cdot \mathrm{m}$.
Acknowledgement: Calculations have been carried out using resources provided by Wroclaw Centre for Networking and Supercomputing (http://wcss.pl), grant No. 444

\section{References}

[1] Kidam K., Hassin M. H., Enhancement of inherent safety and accident prevention in chemical industry by reviewing past accidents, Journal of Chemical and Natural Resources Engineering, 2008 pp 75-86.

[2] Sonnemans PJ, Korvers PM. Accidents in the chemical industry: are they foreseeable? J Loss Prev Process Ind. 2006;19(1):1-12.

[3] Furtek A. Review of hazardous of the pipeline accidents. Centre of Excellence at the Institute of Atomic Energy; 2004.

[4] Jaszak P, Adamek K. Design and analysis of the flange-bolted joint with respect to required tightness and strength. Open Eng. 2019;9(1):338-49.

[5] Fukuoka T, Nomura M, Nishikawa T. Analysis of Thermal and Mechanical Behavior of Pipe Flange Connections by Taking Account of Gasket Compression Characteristics at Elevated Temperature. J Press Vessel Technol. 2012;134(2):021202.

[6] Zylinski B, Buczkowski R. Analysis of bolted joint using the finite element method, Arch. Mech. Eng., Vol. LVII, 2010, Number 3.

[7] Jaszak P. The elastic serrated gasket of the flange bolted joints. Int J Press Vessels Piping. 2019;176:103954.

[8] Jaszak P. A new solution of the semi-metallic gasket increasing tightness level. Open Eng. 2019;9(1):329-37.

[9] Suggs S., Meyer R., Kolb S., Low stress/anti-buckling spiral wound gasket, International Application Published Under The Patent Cooperation Treaty, WO 2017/087643 A2.

[10] Attoui H, Bouzid AH, Waterland JA. Buckling and lateral pressure in spiral wound gaskets, Proceedings of the ASME 2014, Pressure Vesel and Piping Conference 2014, Volume 2: Computer Technology and Bolted Joints, Anaheim, California, USA, July 20-24, (2014), pp. 1-7. https://doi.org/10.1115/PVP2014-28449.

[11] Winter JR, Leon GF. Radially Inward Buckling of Spiral Wound Gaskets. American Society of Mechanical Engineers, Pressure Vessels and Piping Division. PVP-Vol. 1985;98:111-6.

[12] Mueller RT. Recent buckling experiences with spiral wound flexible graphite filled gaskets, American Society of Mechanical Engineers, Pressure Vessels and Piping Division PVP. Volume 326. Computer Technology - Applications and Methodology; 1996. pp. 23-34.

[13] Veiga JC, Cipolatti CF, Kavanagh N, Castro F, Zandona V. Serrated Metal Graphite Faced Gaskets for Ring Joint Flanges, Conference: ASME. Pressure Vessels and Piping Conference; 2012.

[14] Jaszak P., Uszczelnienie metaloplastyczne połączeń kołnierzowośrubowych, Opis patentowy PL 231054 B1.

[15] Jaszak P. Adaptation of a highly compressible elastomeric material model to simulate compressed expanded graphite and its application in the optimization of a graphite-metallic structure'. J Braz Soc Mech Sci Eng. 2020;42(5):224. 